\title{
GENERATION MEAN ANALYSIS OF TWO BREAD WHEAT CROSSES UNDER NORMAL AND LATE SOWING DATE CONDITIONS
}

\author{
YASSER SAYED IBRAHIM KOUBISY
}

Wheat Res. Dep., Field Crops Res. Inst., ARC, Giza, Egypt.

(Manuscript received 31 March 2019)

\begin{abstract}
arents $\left(P_{1} \& P_{2}\right), F_{1}, F_{2}$ and first generation of backcrosses $D\left(\mathrm{BC}_{1} \& \mathrm{BC}_{2}\right)$ of two bread wheat crosses i.e., Sids $12 \times$ Gemmeiza $11\left(C_{1}\right)$ and Misr $2 \times$ Sids $1\left(C_{2}\right)$, were grown in two experiments \{normal sowing date $20^{\text {th }}$ Nov. $(\mathrm{N})$ and late sowing date $20^{\text {th }}$ Dec. (L)\}. This study was conducted at the Experimental Farm of Shandaweel Agric. Res. Station, ARC during 2015/2016, 2016/2017 and 2017/2018 seasons. The study aimed to estimate non-allelic interaction, scaling tests $(A, B, C$ and $D)$, coupled with three and six types of gene action and to determine the adequacy of genetic model controlling the genetic system of the inheritance for seven economic traits of bread wheat. Scaling test showed the presence of non-allelic interactions for all studied taits, except number of spikes/plant in cross 1 under late sowing. The relative importance of dominance and additive effects differ for traits and crosses under normal and late sowing conditions. Dominance effects were generally more important than additive for all studied traits in both crosses under the two sowing dates, except for days to heading in cross 2 under late sowing, number of spikes/plant in cross 1 under late sowing and biological yield in cross 1 under normal sowing. This explains that dominant genes play a major role in the inheritance of these traits beside the additive one. Dominance $\times$ dominance gene interaction was higher in magnitude than additive $\times$ dominance and additive $\times$ additive in most traits, indicating that these traits are greatly affected by dominance and non-allelic interactions. Therefore, it is advisable to delay selection to late segregating generations with increased homozygosity. Positive highly significant heterosis over better parent values was observed for all studied traits in both crosses under the two sowing dates, except for plant height in cross 1 under late sowing and cross 2 under normal sowing, number of kernels/spike in cross 1 under both sowing dates and grain yield/plant in cross 1 under normal sowing. Broad and narrow sense heritabilities and genetic advance ranged from moderate to high in most cases. These results indicated that selection in segregating generations could be effective to develop early maturing lines that have high yielding ability under heat stress conditions (late sowing date).
\end{abstract}

Keywords: Triticum aestivum, six parameters model, gene action, heritability, heterosis.

\section{INTRODUCTION}

In Egypt, wheat is considered the most important cereal crop. Great efforts have been mode by wheat breeders and geneticists to increase it is productivity decrease the gap between production and consumption. Heat stress is one of the 
major limitations facing wheat productivity in arid, semiarid, tropical and subtropical regions (Fischer, 1986). Many studies had confirmed the damaging effect of heat on wheat. El-Gizawy (2009), Seleiman et al. ( 2011), Hamam (2014), EL-Maghraby et al. (2016) and Abd El-Rady (2018) reported that late sowing reduced days to heading, plant height, number of spikes $/ \mathrm{m}^{2}$, number of kernels/spike, 1000-kernel weight, biological yield and final grain yield. Genetic variability for heat tolerance has been found to exist among wheat cultivars and lines. Therefore, wheat breeders and geneticists must continue to develop high yielding cultivars over a wide range of environments with desirable genetic makeup. A detailed understanding of the genetic factors controlling agronomic characteristics is a primary step for breeding programs. Generation mean analysis is a simple estimate but it is one of the most important techniques for estimating main gene effects (dominance and additive) along with their interactions (dominance $\times$ dominance, additive $\times$ additive and additive $\times$ dominance) provided the pattern inheritance of yield and other associated characters. Since, genetic information obtained from multi generations are reliable compared with those based on one generation, therefore, six populations of $P_{1}, P_{2}, F_{1}, F_{2}, B C_{1}$ and $B C_{2}$ are considered the one which may give detailed genetic information for the employed genotypes. Additive dominance model was adequate to explain the type of gene action of grain yield and its components (Bayoumi et al., 2008). Amin (2013) reported that additive dominance model was invalid to explain the inheritance of most studied traits under normal and heat stress conditions. Hamam (2014) and Abd El-Rady (2018) showed that narrow sense heritability estimates in $F_{2}$ were relatively moderate to high under favorable and heat stress conditions for yield and its components. The objectives of this study were to 1 ) determine the nature of gene action controlling yield and its components in two bread wheat crosses under normal and heat stress conditions, 2) estimate heterosis, inbreeding depression, potence ratio, broad and narrow sense heritability and expected genetic advance from selection.

\section{MATERIALS AND METHODS}

This study was carried out at the Experimental Farm of Shandaweel Agric. Res. Station, ARC., Egypt, during 2015/2016, 2016/2017 and 2017/2018 seasons. Four bread wheat (Triticum aestivum ) cultivars representing a wide range of diversity for several agronomic traits were used as parents to obtain the following two crosses; Cross $1=$ Sids 12 x Gemmeiza 11 and Cross $2=$ Misr 2 x Sids 1 (Table 1). 
Table 1. Pedigree and selection history of cultivars used in the two bread wheat crosses.

\begin{tabular}{|l|l|l|l|}
\hline \multicolumn{2}{|c|}{ Cross } & \multicolumn{1}{|c|}{ Parent } & \multicolumn{1}{c|}{ Pedigree and selection history } \\
\hline \multirow{4}{*}{ Cross 1 } & P1 & Sids 12 & $\begin{array}{l}\text { BUC//7C/ALD/5/MAYA74/ON//1160- } \\
\text { 147/3/BBGLL/4/HAT"S"/6/MAYA/VUL//CMH74A.630/4*SX.SD7096- } \\
\text { 4SD-1SD-1SD-0SD }\end{array}$ \\
\cline { 2 - 5 } & P2 & Gemmeiza 11 & $\begin{array}{l}\text { BOW"S"/KVZ"S"//7C/SERI-82/3/GIZA 168/SAKHA 61 } \\
\text { GM7892-2GM-1GM-2GM-1GM-0GM }\end{array}$ \\
\hline \multirow{3}{*}{ Cross 2 } & P1 & Misr 2 & $\begin{array}{l}\text { SKAUZ/BAV92. } \\
\text { CMSS96M03611S-1M-0105Y-33M-0Y-0S }\end{array}$ \\
\cline { 2 - 5 } & P2 & Sids 1 & $\begin{array}{l}\text { HD2172 / PAVON "S" // 1158.57/ MAYA74"S" } \\
\text { SD46-4SD-2SD-1SD-0SD }\end{array}$ \\
\hline
\end{tabular}

Table 2. Mean maximum and minimum air temperatures $\left({ }^{\circ} \mathrm{C}\right)$ during $2015 / 2016,2016 / 2017$ and $2017 / 2018$ growing seasons.

\begin{tabular}{|c|l|c|c|c|c|c|c|c|}
\hline Season & Month & November & December & January & February & March & April & May \\
\hline \multirow{2}{*}{$2015 / 2016$} & Max. & 27 & 21 & 19 & 25 & 28 & 36 & 38 \\
\cline { 2 - 9 } & Min. & 15 & 9 & 7 & 10 & 15 & 19 & 23 \\
\hline \multirow{2}{*}{$2016 / 2017$} & Max. & 28 & 21 & 20 & 21 & 26 & 33 & 38 \\
\cline { 2 - 9 } & Min. & 15 & 8 & 7 & 7 & 12 & 18 & 22 \\
\hline \multirow{2}{*}{$2017 / 2018$} & Max. & 26 & 21 & 15 & 22 & 29 & 32 & 36 \\
\cline { 2 - 9 } & Min. & 14 & 13 & 11 & 13 & 19 & 21 & 23 \\
\hline
\end{tabular}

In the first growing season (2015/2016), two crosses were performed using the four wheat cultivars to produce $F_{1}$ hybrid seed. In the second growing season (2016/2017), the $F_{1}$ of each cross was crossed to its respective parents to produce $\mathrm{BC}_{1}\left(\mathrm{~F}_{1} \times \mathrm{P}_{1}\right)$ and $\mathrm{BC}_{2}\left(\mathrm{~F}_{1} \times \mathrm{P}_{2}\right)$. At the same time, the other $\mathrm{F}_{1}$ plants were selfed to produce $F_{2}$ seed. In the third growing season (2017/2018), the six populations, i.e., $\mathrm{P}_{1}, \mathrm{P}_{2}, \mathrm{~F}_{1}, \mathrm{~F}_{2}, \mathrm{BC}_{1}$ and $\mathrm{BC}_{2}$ of the two wheat crosses were sown under two sowing dates in two separate field experiments in a randomized complete block design with three replications. The first sowing date was $20^{\text {th }}$ Nov., recommended, and the second one was $20^{\text {th }}$ Dec., (heat stress). Each replicate consisted of 12 rows, one row for each of $P_{1}, P_{2}$ and $F_{1}, 5$ rows for $F_{2}$ and 2 rows for each of $B C_{1}$ and $B C_{2}$ for each cross with total of 24 rows as well as two border rows. Each row was $2.0 \mathrm{~m}$ long and $30 \mathrm{~cm}$ apart with $10 \mathrm{~cm}$ plant spacing. All other Agricultural practices were applied as recommended for wheat planting. Data were recorded on 10 individual guarded plants for each $\mathrm{P}_{1}, \mathrm{P}_{2}$ and $\mathrm{F}_{1} ; 75$ plants of $\mathrm{F}_{2}$ and 20 plants of $\mathrm{BC}_{1}$ and $\mathrm{BC}_{2}$ in each replicate. The studied traits were: 1 - days to $50 \%$ heading, 2- plant height $(\mathrm{cm}), 3-$ number of spikes/plant, 4- number of kernels/spike, 5- 100-kernel weight (g) 6- biological yield/plant ( $\mathrm{g}$ ) and 7- grain yield/plant $(\mathrm{g})$. 


\section{Statistical and genetic analysis:}

Analysis of variance and character mean comparisons were performed using M-STAT statistical program. The scaling test was applied to detect the presence or absence of non-allelic interactions and their types as outlined by Mather (1949).
$A=\overline{2 B} C_{1}-P_{1}-F_{1}$
$\mathrm{V}(\mathrm{A})=4 \mathrm{~V}\left(\overline{\mathrm{BC}}_{1}\right)+\mathrm{V}\left(\overline{\mathrm{P}}_{1}\right)+\mathrm{V}\left(\mathrm{F}_{1}\right)$
$\mathrm{B}=2 \overline{\mathrm{BC}}_{2}-\overline{\mathrm{P}}_{2}-\overline{\mathrm{F}}_{1}$
$\mathrm{V}(\mathrm{B})=4 \mathrm{~V}\left(\overline{\mathrm{BC}}_{2}\right)+\mathrm{V} \overline{\left(\mathrm{P}_{2}\right)}+\mathrm{V} \overline{\left(\overline{\mathrm{F}_{1}}\right)}$
$\mathrm{C}=\overline{4} \mathrm{~F}_{2}-\overline{2} \mathrm{~F}_{1}-\overline{\mathrm{P}_{1}}-\overline{\mathrm{P}_{2}}$
$\mathrm{V}(\mathrm{C})=16 \mathrm{~V}\left(\mathrm{~F}_{2}\right)+4 \mathrm{~V}\left(\mathrm{~F}_{1}\right)+\mathrm{V}\left(\overline{\left.\mathrm{P}_{1}\right)}+\mathrm{V}\left(\overline{\left.\mathrm{P}_{2}\right)}\right.\right.$
$\mathrm{D}=\overline{2}_{2}-\overline{\mathrm{BC}}_{1}-\overline{\mathrm{BC}}_{2}$
$\mathrm{V}(\mathrm{D})=\overline{4 \mathrm{VF}_{2}}+\overline{\mathrm{VBC}}_{1}+\overline{\mathrm{VBC}}_{2}$

The standard error (SE) of $A, B, C$ and $D$ was achieved by taking the square root of their respective variances. T-test was calculated by dividing the calculated values of $A, B, C$ and $D$ on their respective standard error. The significance of any one of these scales is taken to indicate the presence of epistasis. In the presence of epistasis various gene effects were estimated using six parameters genetic model of Jinks and Jones (1958) and Hayman (1958).

$\mathrm{m}=$ mean effect $=\overline{\mathrm{F}_{2}}$

$\mathrm{d}=$ additive effect $=\overline{\mathrm{BC}}-\overline{\mathrm{BC}_{2}}$

$\mathrm{h}=$ dominance effect $=\overline{\mathrm{F}_{1}}-4 \overline{\mathrm{F}}_{2}-0 . \overline{5 \mathrm{P}_{1}}-0 . \overline{5 \mathrm{P}_{2}}+2 \overline{\mathrm{BC}}_{1}+2 \overline{\mathrm{BC}}_{2}$

$\mathrm{i}=$ Additive $\times$ Additive gene interaction $=2 \overline{\mathrm{BC}_{1}}+2 \overline{\mathrm{BC}_{2}}-4 \overline{\mathrm{F}_{2}}$

$\mathrm{j}=$ Additive $x$ Dominance gene interaction $=\mathrm{BC}_{1}-0.5 \overline{\mathrm{P}_{1}}-\overline{\mathrm{BC}_{2}}+0 . \overline{\mathrm{P}}$

$\mathrm{I}=$ Dominance $x$ Dominance gene action $=\overline{\mathrm{P}}_{1}+\overline{\mathrm{P}_{2}}+\overline{2 \mathrm{~F}_{1}}+\overline{4 \mathrm{~F}_{2}}-4 \overline{\mathrm{BC}}_{1}-4 \overline{\mathrm{BC}}_{2}$

The variance values in this concern were obtained as follows:

$$
\begin{aligned}
& \mathrm{V}_{\mathrm{m}}=\mathrm{V}\left(\mathrm{F}_{2}\right) \\
& \mathrm{V}_{\mathrm{d}}=\mathrm{V}\left(\overline{\left.\mathrm{BC}_{1}\right)}+\mathrm{V}\left(\overline{\mathrm{BC}} \overline{\mathrm{C}}_{2}\right)\right. \\
& V_{h}=V \overline{\left(F_{1}\right)}+16 V\left(\overline{\left.F_{2}\right)}+0.25 V \overline{\left(\mathrm{P}_{1}\right)}+\underline{0} .25 \mathrm{~V}\left(\mathrm{P}_{2}\right)+4 \mathrm{~V}\left(\mathrm{BC}_{1}\right)+4 \mathrm{~V}\left(\overline{\mathrm{BC}_{2}}\right)\right. \\
& \mathrm{V}_{\mathrm{i}}=4 \mathrm{~V}\left(\underline{\mathrm{BC}_{1}}\right)+4 \mathrm{~V}\left(\mathrm{BC}_{2}\right)+16 \mathrm{~F}_{2} \\
& V_{j}=V\left(\overline{\left.B C_{1}\right)}+0.25 V\left(\overline{P_{1}}\right)+V\left(\overline{B C_{2}}\right)+0.25 \overline{V\left(P_{2}\right)}\right. \\
& V_{j}=V\left(\overline{B C_{1}}\right)+0.25 \overline{\left(P_{1}\right)}+V\left(\overline{B C_{2}}\right)+0.25 V\left(\overline{\left.P_{2}\right)}\right. \\
& V_{1}=V\left(P_{1}\right)+V\left(P_{2}\right)+4 V\left(F_{1}\right)+16 V\left(F_{2}\right)+16 V\left(B_{1}\right)+16 V\left(B_{2}\right)
\end{aligned}
$$

The significance of the genetic components were tested using the $t$ test, where $\pm t=$ effect/ (variance effect ) $)^{1 / / 2}$

Inbreeding depression was estimated as the average percentage decrease of the $F_{2}$ from the $F_{1}$ as follows:

$$
\text { (I.D \%) }=\left(\overline{F_{1}}-\overline{F_{2}} / \overline{F_{1}}\right) \times 100
$$

Potence ratio $(P)$, was estimated by using the following equation:

$P=\left(F_{1}-M P\right) / 1 / 2\left(P_{2}-P_{1}\right)$ where: $F_{1}$ : first generation mean, $P_{1}$ : the mean of the lower parent, $\mathrm{P}_{2}$ : the mean of the higher parent, and MP: the mid-parent values = $1 / 2\left(P_{1}+P_{2}\right)$. 
Stress tolerance index (STI) for grain yield was computed according to Farshadfar, et al. (2001), as follow: STI $=$ Yp x Ys $/(Y p)^{2} \times 100$

where, Yp grain yield under normal conditions, Ys grain yield under stress conditions.

The genetic components of variance were calculated by the formulas of $F_{2}$ variance were obtained according to Mather and Jinks (1982) as:

$E$ (environmental variance $)=1 / 3\left(V_{P 1}+V_{P 2}+V_{F 1}\right)$

$\mathrm{D}$ (additive variance) $=4 \mathrm{~V}_{\mathrm{F} 2}-2\left(\mathrm{~V}_{\mathrm{BC} 1}+\mathrm{V}_{\mathrm{BC} 2}\right)$

$\mathrm{H}$ (dominance variance $)=4\left(\mathrm{~V}_{\mathrm{F} 2}-1 / 2 \mathrm{~V}_{\mathrm{D}}-\mathrm{V}_{\mathrm{E}}\right)$

Broad-sense $\left(h^{2}\right.$ b.s $)$ and narrow-sense $\left(h^{2}{ }_{n . s}\right)$ heritability were estimated using Warner (1952) formulas:

$h^{2} b . s=\left[V_{F 2}-\left(V_{p 1}+V_{p 2}+F_{1}\right) / 3\right] / V_{F 2}$

$\mathrm{h}^{2}$.s $=\left[2 \mathrm{~V}_{\mathrm{F} 2}-\left(\mathrm{V}_{\mathrm{BC} 1}+\mathrm{V}_{\mathrm{BC} 2}\right)\right] / \mathrm{V}_{\mathrm{F} 2}$

Genetic advance was computed according to Johnson et al. (1955) with selection intensity of $K=5 \%$ (2.06) for all characters as follows:

$\mathrm{G} . \mathrm{S}=\mathrm{K} \times\left(\sigma^{2} \mathrm{~F}_{2}\right)^{1 / 2} \times \mathrm{h}^{2} \mathrm{n}$. and $(\mathrm{G} . \mathrm{S} \%)=\left(\mathrm{G} . \mathrm{S} / \overline{\mathrm{F}}_{2}\right) \times 100$

Heterosis was expressed as the deviation of $F_{1}$ generation from the midparents or better parent average values as follows:

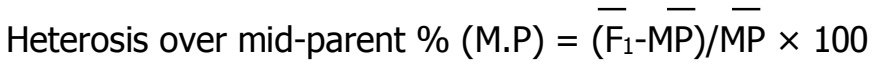

Heterosis over the better-parent $\%(\mathrm{BP})=\left(\overline{\mathrm{F}_{1}}-\overline{\mathrm{BP}}\right) / \overline{\mathrm{BP}} \times 100$

\section{RESULTS AND DISCUSSION}

\section{Mean performance:}

Average of the seven characters for the six populations $P_{1}, P_{2}, F_{1}, F_{2}, B C_{1}$ and $\mathrm{BC}_{2}$ in the two bread wheat crosses under the two sowing dates are given in Table 3. Significant differences were found among the six generations for all the studied traits under the two environmental conditions, indicating the existence of genetic variation for these traits in the studied materials. The $F_{1}$ mean values surpassed the mid values of the two parental means for all studied traits in the two crosses under both recommended and late sowings, except days to heading in cross 2 under normal sowing and 100-kernel weight in cross 1 under late sowing conditions, indicating the prevalence of heterotic and dominance effects controlling these traits. The $F_{1}$ means exceeded the better parent for all studied traits in the two crosses under both sowing environments except, days to heading in cross 1 under both sowing dates and in cross 2 under normal sowing date, 100-kernel weight in cross 1 under late sowing conditions and in cross 2 under both sowing dates, plant height in cross 2 under both sowing dates, and number of kernels/spike in cross 2 under late sowing, indicating the presence of over dominance. The $F_{2}$ population mean performance values were less 
than $F_{1}$ for all the studied traits in two crosses under both environments, except plant height in cross 1 under normal conditions, showing the importance of non-additive components of genetic variance for these characters. However, average value of $\mathrm{BC} 1$ and $\mathrm{BC}_{2}$ progenies of the two crosses varied under normal and late sowing dates conditions and each tended toward the mean of its recurrent parent. These results agreed with those reported by Tammam (2005), El-Aref et al. (2011), Amin (2013), Hamam (2014), Said (2014), Kumar et al. (2017) and Abd El-Rady (2018).

Stress tolerance index (STI) (Table 3) showed that the $\mathrm{BC}_{2}$ had the highest value of heat tolerance (96.35\%) followed by $F_{2}, F_{1}, P_{1}, P_{2}$ and $B C_{1}$ which had $95.24 \%, 93.14 \%, 90.55 \%, 89.92 \%$ and $78.36 \%$, respectively in cross 1 . While in cross 2 , the $F_{1}$ hybrid had the highest value of STI (84.69\%) followed by $\mathrm{P}_{1}(80.37 \%)$, $\mathrm{F}_{2}$ population $(76.54), \mathrm{P}_{2}(76.34 \%), \mathrm{BC}_{2}(74.69 \%)$ and $\mathrm{BC}_{1}(60.81 \%)$. These results indicated that selection in the segregating populations for grain yield development under late sowing conditions could be effective to develop high yielding lines that have tolerance to heat stress. Similar results were obtained by Amin (2013) and Abd El-Rady (2018).

\section{Gene effects:}

The results of $A, B, C$, and $D$ scaling tests for the two bread wheat crosses under the two environmental conditions (Table 4) revealed the significance of any of these tests in the two crosses under both environments, except for number of spikes/plant in cross 1 at late sowing conditions. These findings indicated that the presences of non allelic gene interaction and dominance-additive model are inadequate for explaining the inheritance of these traits. Meanwhile, the scaling test estimates for number of spikes/plant in cross 1 under late sowing was insignificant indicated the absence of non-allelic interactions and the adequacy of the additive-dominance model to explain the type of gene action for these traits. These results are in accordance with those reported by Tammam (2005), Moussa (2010), El-Aref et al. (2011), Zaazaa et al. (2012), Amin (2013), Abd El-Rahman (2013), Hamam (2014), El-Hawary (2016), Kumar et al. (2017) and Abd El-Rady (2018).

Data in Table 5 show the six parameters of gene effects for two wheat crosses under two environments. The mean effect $(\mathrm{m})$ which reflects the contribution due the overall mean plus the locus effects and interactions of the fixed loci were highly significant for all studied characters in the two crosses under normal and late sowings, indicating that these characters are quantitatively inherited. The same results were obtained by Moussa (2010), Amin (2013), Hamam (2014), Bilgin et al. (2016), El-Hawary (2016) and Abd El-Rady (2018). 
Table 3. Mean performance \pm standard error of $P_{1}, P_{2}, F_{1}, F_{2}, B C_{1}$ and $B C_{2}$ populations of two bread wheat crosses for the studied traits under normal $(\mathrm{N})$ and late sowing $(\mathrm{L})$ conditions.

\begin{tabular}{|c|c|c|c|c|c|c|c|c|c|}
\hline \multirow{2}{*}{ Trait } & \multirow{2}{*}{ Cross } & \multirow{2}{*}{$\begin{array}{l}\text { Sowing } \\
\text { date }\end{array}$} & \multicolumn{6}{|c|}{ Generation } & \multirow{2}{*}{$\begin{array}{r}\text { L.S.D } \\
0.05 \\
\end{array}$} \\
\hline & & & $P_{1}$ & $P_{2}$ & $\mathrm{~F}_{1}$ & $F_{2}$ & $\mathrm{BC}_{1}$ & $\mathrm{BC}_{2}$ & \\
\hline \multirow{4}{*}{$\begin{array}{l}\text { Days to } 50 \% \\
\text { heading }\end{array}$} & \multirow{2}{*}{$\mathrm{C}_{1}$} & $\mathrm{~N}$ & $87.00 \pm 0.26$ & $94.00 \pm 0.25$ & $92.00 \pm 0.21$ & $91.00 \pm 0.13$ & $91.00 \pm 0.23$ & $89.50 \pm 0.23$ & 5.91 \\
\hline & & $\mathrm{L}$ & $85.00 \pm 0.26$ & $88.00 \pm 0.26$ & $86.50 \pm 0.26$ & $83.00 \pm 0.14$ & $86.00 \pm 0.21$ & $86.60 \pm 0.25$ & 3.21 \\
\hline & \multirow{2}{*}{$\mathrm{C}_{2}$} & $\mathrm{~N}$ & $97.00 \pm 0.26$ & $94.00 \pm 0.26$ & $93.00 \pm 0.26$ & $91.13 \pm 0.21$ & $98.00 \pm 0.29$ & $90.5 \pm 0.35$ & 2.34 \\
\hline & & $\mathrm{L}$ & $87.00 \pm 0.25$ & $84.00 \pm 0.27$ & $89.00 \pm 0.28$ & $83.13 \pm 0.16$ & $83.00 \pm 0.26$ & $80.50 \pm 0.27$ & 3.35 \\
\hline \multirow{4}{*}{$\begin{array}{l}\text { Plant height, } \\
\mathrm{cm} .\end{array}$} & \multirow{2}{*}{$\mathrm{C}_{1}$} & $\mathrm{~N}$ & $90.40 \pm 0.74$ & $100.40 \pm 0.63$ & $102.93 \pm 0.81$ & $102.47 \pm 0.48$ & $92.00 \pm 0.92$ & $99.01 \pm 0.81$ & 6.41 \\
\hline & & $\mathrm{L}$ & $86.00 \pm 0.64$ & $90.53 \pm 0.66$ & $91.53 \pm 0.84$ & $85.00 \pm 0.41$ & $84.00 \pm 0.73$ & $89.11 \pm 0.75$ & 3.06 \\
\hline & \multirow{2}{*}{$\mathrm{C}_{2}$} & $\mathrm{~N}$ & $101.53 \pm 0.69$ & $106.60 \pm 0.56$ & $105.07 \pm 0.78$ & $99.36 \pm 0.39$ & $100.80 \pm 0.72$ & $99.77 \pm 0.72$ & 2.80 \\
\hline & & $\mathrm{L}$ & $91.03 \pm 0.71$ & $98.21 \pm 0.91$ & $95.53 \pm 0.91$ & $91.42 \pm 0.63$ & $94.03 \pm 0.20$ & $92.10 \pm 0.21$ & 3.63 \\
\hline \multirow{4}{*}{$\begin{array}{l}\text { Number of } \\
\text { spikes / plant }\end{array}$} & \multirow{2}{*}{$\mathrm{C}_{1}$} & $\mathrm{~N}$ & $6.69 \pm 0.36$ & $6.00 \pm 0.24$ & $7.30 \pm 0.30$ & $6.11 \pm 0.17$ & $7.13 \pm 0.31$ & $6.40 \pm 0.24$ & 1.33 \\
\hline & & $\mathrm{L}$ & $6.20 \pm 0.25$ & $5.77 \pm 0.13$ & $6.25 \pm 0.17$ & $5.80 \pm 0.12$ & $5.83 \pm 21$ & $5.90 \pm 0.17$ & 0.48 \\
\hline & \multirow{2}{*}{$\mathrm{C}_{2}$} & $\mathrm{~N}$ & $8.27 \pm 0.34$ & $7.79 \pm 0.32$ & $8.80 \pm 0.37$ & $6.48 \pm 0.15$ & $8.11 \pm 0.27$ & $7.17 \pm 0.27$ & 1.21 \\
\hline & & $\mathrm{L}$ & $7.73 \pm 0.31$ & $6.40 \pm 0.21$ & $8.13 \pm 0.18$ & $6.60 \pm 0.12$ & $7.70 \pm 0.20$ & $7.77 \pm 0.21$ & 2.01 \\
\hline \multirow{4}{*}{$\begin{array}{c}\text { Number of } \\
\text { Kernels / spike }\end{array}$} & \multirow{2}{*}{$\mathrm{C}_{1}$} & $\mathrm{~N}$ & $30.22 \pm 0.87$ & $35.61 \pm 1.09$ & $36.57 \pm 1.12$ & $29.19 \pm 0.63$ & $29.20 \pm 1.01$ & $33.75 \pm 1.08$ & 4.76 \\
\hline & & $\mathrm{L}$ & $28.65 \pm 1.08$ & $30.44 \pm 1.33$ & $31.47 \pm 1.87$ & $28.00 \pm 0.64$ & $29.22 \pm 1.09$ & $31.18 \pm 1.20$ & 2.46 \\
\hline & \multirow{2}{*}{$\mathrm{C}_{2}$} & $\mathrm{~N}$ & $35.86 \pm 1.06$ & $39.56 \pm 1.22$ & $41.54 \pm 1.49$ & $34.10 \pm 0.77$ & $33.13 \pm 1.33$ & $35.15 \pm 1.26$ & 2.34 \\
\hline & & L & $30.93 \pm 1.52$ & $36.63 \pm 1.47$ & $35.03 \pm 1.42$ & $29.94 \pm 0.76$ & $30.52 \pm 1.32$ & $28.67 \pm 1.26$ & 1.55 \\
\hline \multirow{4}{*}{$\begin{array}{l}\text { 100-kernel } \\
\text { weight, g. }\end{array}$} & \multirow{2}{*}{$\mathrm{C}_{1}$} & $\mathrm{~N}$ & $5.43 \pm 0.06$ & $5.00 \pm 0.04$ & $5.50 \pm 0.08$ & $4.85 \pm 0.05$ & $5.11 \pm 0.08$ & $4.90 \pm 0.08$ & 0.70 \\
\hline & & L & $4.70 \pm 0.07$ & $4.11 \pm 0.07$ & $4.31 \pm 0.06$ & $3.95 \pm 0.03$ & $4.40 \pm 0.06$ & $4.00 \pm 0.06$ & 0.65 \\
\hline & \multirow{2}{*}{$\mathrm{C}_{2}$} & $\mathrm{~N}$ & $4.25 \pm 0.05$ & $4.89 \pm 0.06$ & $4.76 \pm 0.06$ & $3.90 \pm 0.03$ & $4.28 \pm 0.05$ & $4.73 \pm 0.04$ & 0.78 \\
\hline & & $\mathrm{L}$ & $4.20 \pm 0.05$ & $3.75 \pm 0.06$ & $4.01 \pm 0.05$ & $3.60 \pm 0.04$ & $3.97 \pm 0.06$ & $3.96 \pm 0.06$ & 0.49 \\
\hline \multirow{4}{*}{$\begin{array}{l}\text { Biological yield } \\
\text { /plant, g. }\end{array}$} & \multirow{2}{*}{$\mathrm{C}_{1}$} & $\mathrm{~N}$ & $40.33 \pm 1.94$ & $42.27 \pm 1.82$ & $45.87 \pm 1.84$ & $39.33 \pm 1.20$ & $37.20 \pm 2.03$ & $39.20 \pm 1.77$ & 2.41 \\
\hline & & $\mathrm{L}$ & $39.87 \pm 0.66$ & $41.60 \pm 1.46$ & $44.60 \pm 0.93$ & $38.35 \pm 0.97$ & $40.53 \pm 1.45$ & $41.53 \pm 1.51$ & 1.64 \\
\hline & \multirow{2}{*}{$\mathrm{C}_{2}$} & $\mathrm{~N}$ & $42.27 \pm 0.83$ & $54.60 \pm 0.89$ & $56.27 \pm 0.78$ & $40.70 \pm 0.60$ & $38.30 \pm 1.00$ & $48.47 \pm 0.92$ & 2.28 \\
\hline & & $\mathrm{L}$ & $34.73 \pm 1.42$ & $38.60 \pm 1.46$ & $49.93 \pm 1.53$ & $30.79 \pm 0.76$ & $32.33 \pm 1.33$ & $35.53 \pm 1.26$ & 2.39 \\
\hline \multirow{4}{*}{$\begin{array}{l}\text { Grain yield/ } \\
\text { plant, g. }\end{array}$} & \multirow{2}{*}{$\mathrm{C}_{1}$} & $\mathrm{~N}$ & $11.43 \pm 0.50$ & $12.57 \pm 0.55$ & $12.74 \pm 0.64$ & $10.65 \pm 0.41$ & $11.60 \pm 0.76$ & $10.79 \pm 0.66$ & 1.10 \\
\hline & & $\mathrm{L}$ & $10.35 \pm 0.45$ & $11.30 \pm 0.36$ & $11.87 \pm 0.57$ & $10.57 \pm 0.25$ & $9.09 \pm 0.47$ & $10.40 \pm 0.41$ & 2.07 \\
\hline & \multirow{2}{*}{$\mathrm{C}_{2}$} & $\mathrm{~N}$ & $11.97 \pm 0.68$ & $14.85 \pm 0.72$ & $17.41 \pm 0.82$ & $11.93 \pm 0.48$ & $15.77 \pm 0.80$ & $13.87 \pm 0.72$ & 1.35 \\
\hline & & $\mathrm{L}$ & $9.62 \pm 0.42$ & $11.33 \pm 0.53$ & $14.74 \pm 0.68$ & $9.13 \pm 0.27$ & $9.59 \pm 0.50$ & $10.36 \pm 0.44$ & 1.30 \\
\hline STI\% & \multicolumn{2}{|c|}{$C_{1}$} & 90.55 & 89.92 & 93.14 & 95.24 & 78.36 & 96.35 & \\
\hline STI\% & \multicolumn{2}{|c|}{$\mathrm{C}_{2}$} & 80.37 & 76.34 & 84.69 & 76.54 & 60.81 & 74.69 & \\
\hline
\end{tabular}

$\mathrm{STI}=$ Stress tolerance index for grain yield/plant, Cross $1=($ Sids $12 \times$ Gemmeiza 11), Cross $2=($ Misr $2 \times$ Sids 1$)$ 

SOWING DATE CONDITIONS

Table 4. Scaling test parameters A, B, C and D of two bread wheat crosses for all studied traits under normal $(N)$ and late sowing (L) conditions.

\begin{tabular}{|c|c|c|c|c|c|}
\hline \multirow{2}{*}{ Cross } & \multirow{2}{*}{$\begin{array}{c}\text { Sowing } \\
\text { date }\end{array}$} & \multicolumn{4}{|c|}{ Scaling test } \\
\hline & & $\mathbf{A}$ & B & C & D \\
\hline \multicolumn{6}{|c|}{ Days to heading } \\
\hline \multirow{2}{*}{$\mathrm{C}_{1}$} & $\mathbf{N}$ & $3.00 * * \pm 0.57$ & $-7.00 * * \pm 0.57$ & $-5.21 * * \pm 0.76$ & $-0.61 \pm 0.42$ \\
\hline & $\mathbf{L}$ & $-2.00 * * \pm 0.57$ & $-3.80 * * \pm 0.62$ & $-19.00 * * \pm 0.85$ & $-6.60 * * \pm 0.43$ \\
\hline \multirow{2}{*}{$\mathrm{C}_{2}$} & $\mathbf{N}$ & $6.00 * * \pm 0.70$ & $-6.00 * * \pm 0.79$ & $-12.47 * * \pm 1.05$ & $-6.23 * * \pm 0.62$ \\
\hline & $\mathbf{L}$ & $-10 * * \pm 0.64$ & $-12.00 * * \pm 0.65$ & $-16.48 * * \pm 0.91$ & $2.76 * * \pm 0.49$ \\
\hline \multicolumn{6}{|c|}{ Plant height } \\
\hline \multirow{2}{*}{$\mathrm{C}_{1}$} & $\mathbf{N}$ & $-9.33 * * \pm 2.14$ & $-5.33 * * \pm 1.91$ & $13.22 * * \pm 2.69$ & $13.94 * * \pm 1.55$ \\
\hline & $\mathbf{L}$ & $-9.53 * * \pm 1.80$ & $-4.07 * \pm 1.83$ & $-19.60 * * \pm 2.54$ & $-3.00 * \pm 1.33$ \\
\hline \multirow{2}{*}{$\mathrm{C}_{2}$} & $\mathbf{N}$ & $-5.00 * * \pm 1.78$ & $-12.13^{* *} \pm 1.73$ & $-20.81 * * \pm 2.39$ & $-1.84 \pm 1.28$ \\
\hline & $\mathbf{L}$ & $1.50 \pm 2.39$ & $-9.54 * * \pm 2.31$ & $-14.64 * * \pm 3.32$ & $-3.30 \pm 1.89$ \\
\hline \multicolumn{6}{|c|}{ Number of spikes/plant } \\
\hline \multirow{2}{*}{$\mathrm{C}_{1}$} & $\mathbf{N}$ & $0.28 \pm 0.78$ & $-0.50 \pm 0.62$ & $-2.85^{* *} \pm 1.00$ & $-1.31 * \pm 0.52$ \\
\hline & $\mathbf{L}$ & $-0.79 \pm 0.52$ & $-0.22 \pm 0.41$ & $--1.27 \pm 0.65$ & $-0.13 \pm 0.36$ \\
\hline \multirow{2}{*}{$\mathrm{C}_{2}$} & $\mathbf{N}$ & $-0.85 \pm 0.73$ & $-2.25^{* *} \pm 0.73$ & $-7.74 * * \pm 1.05$ & $-2.32 * * \pm 0.48$ \\
\hline & $\mathbf{L}$ & $-0.46 \pm 0.54$ & $1.00 * \pm 0.50$ & $-4.01 * * \pm 0.71$ & $-2.28 * * \pm 0.38$ \\
\hline \multicolumn{6}{|c|}{ Number of kernels/spike } \\
\hline \multirow{2}{*}{$\mathbf{C}_{1}$} & $\mathbf{N}$ & $-8.41 * * \pm 2.46$ & $-4.67 * \pm 2.67$ & $-22.21 * * \pm 3.95$ & $-4.56 * \pm 1.95$ \\
\hline & $\mathbf{L}$ & $-1.68 \pm 3.07$ & $0.45 \pm 3.32$ & $-10.03 * \pm 4.85$ & $-4.40 * \pm 2.06$ \\
\hline \multirow{2}{*}{$\mathrm{C}_{2}$} & $\mathbf{N}$ & $-11.14^{* *} \pm 3.22$ & $-10.80 * * \pm 3.18$ & $-22.12 * * \pm 4.57$ & $-0.09 \pm 2.39$ \\
\hline & $\mathbf{L}$ & $-11.74 * * \pm 3.37$ & $-14.32 * * \pm 3.25$ & $-17.86^{* *} \pm 6.67$ & $4.10 \pm 2.38$ \\
\hline \multicolumn{6}{|c|}{ 100-kernel weight } \\
\hline \multirow{2}{*}{$\mathbf{C}_{1}$} & $\mathbf{N}$ & $-0.71 * * \pm 0.19$ & $-0.70 * * \pm 0.19$ & $-2.03 * * \pm 0.28$ & $-0.31 * \pm 0.15$ \\
\hline & $\mathbf{L}$ & $-0.21 \pm 0.15$ & $-0.42 * * \pm 0.16$ & $-1.62 * * \pm 0.22$ & $-0.50 * * \pm 0.11$ \\
\hline \multirow{2}{*}{$\mathrm{C}_{2}$} & $\mathbf{N}$ & $-0.45^{* *} \pm 0.13$ & $-0.20 \pm 0.12$ & $-3.05^{* *} \pm 0.18$ & $-1.20 * * \pm 0.08$ \\
\hline & $\mathbf{L}$ & $-0.27 \pm 015$ & $0.16 \pm 0.13$ & $-1.58 * * \pm 0.19$ & $-0.74 * * \pm 0.11$ \\
\hline \multicolumn{6}{|c|}{ Biological yield/plant } \\
\hline \multirow{2}{*}{$C_{1}$} & $\mathbf{N}$ & $-11.80 * \pm 4.86$ & $-9.73 * \pm 4.39$ & $-17.02 * \pm 6.61$ & $2.26 \pm 3.61$ \\
\hline & $\mathbf{L}$ & $-3.41 \pm 3.12$ & $-3.14 \pm 3.48$ & $-17.27 * * \pm 4.59$ & $-5.36 * \pm 2.85$ \\
\hline \multirow{2}{*}{$\mathrm{C}_{2}$} & $\mathbf{N}$ & $-21.93 * * \pm 2.31$ & $-13.93 * * \pm 2.18$ & $-46.60 * * \pm 3.11$ & $-5.37 * * \pm 1.81$ \\
\hline & $\mathbf{L}$ & $-20.01 * * \pm 3.38$ & $-17.47 * * \pm 3.29$ & $-50.04 * * \pm 4.77$ & $-6.28 * * \pm 2.38$ \\
\hline \multicolumn{6}{|c|}{ Grain yield/plant } \\
\hline \multirow{2}{*}{$\mathbf{C}_{1}$} & $\mathbf{N}$ & $-0.97 \pm 1.73$ & $-3 . .72 * \pm 1.57$ & $-6.88 * * \pm 2.22$ & $-1.09 \pm 1.31$ \\
\hline & $\mathbf{L}$ & $-4.04 * * \pm 1.18$ & $-2.37 * \pm 1.06$ & $-3.11 \pm 1.61$ & $1.65 * \pm 0.79$ \\
\hline \multirow{2}{*}{$\mathrm{C}_{2}$} & $\mathbf{N}$ & $2.16 \pm 1.93$ & $-4.52 * \pm 1.80$ & $-13.92 * * \pm 2.70$ & $-5.78 * * \pm 1.44$ \\
\hline & $\mathbf{L}$ & $-5.19 * * \pm 1.28$ & $-5.36 * * \pm 1.23$ & $-13.92 * * \pm 1.86$ & $-1.69 * \pm 0.86$ \\
\hline
\end{tabular}

$* \& * *$ Significant and high Significant at $0.05 \& 0.01$ level of probabilities, respectively. 
Table 5. Types of gene action using generation means \pm standard error for all studied traits of two bread wheat crosses under normal $(\mathrm{N})$ and late sowing dates $(\mathrm{L})$ conditions.

\begin{tabular}{|c|c|c|c|c|c|c|c|c|c|}
\hline \multirow{2}{*}{ Trait } & \multirow{2}{*}{ Cross } & \multirow{2}{*}{$\begin{array}{l}\text { Sowing } \\
\text { date }\end{array}$} & \multicolumn{6}{|c|}{ Gene effect } & \multirow{2}{*}{ 요 } \\
\hline & & & Mean (m) & Additive (d) & Dominance (h) & Additive $\mathrm{x}$ & Additive $\mathrm{x}$ & Dominance $\mathrm{x}$ & \\
\hline \multirow{4}{*}{$\begin{array}{l}\text { Days to } \\
\text { heading }\end{array}$} & \multirow{2}{*}{ C1 } & $\mathbf{N}$ & $89.95 * * \pm 0.13$ & $1.50 * * \pm 0.33$ & $2.71 * * \pm 0.88$ & $1.21 \pm 0.84$ & $5.00 * * \pm 0.37$ & $2.79 \pm 1.51$ & $\mathrm{C}$ \\
\hline & & $\mathbf{L}$ & $83.00 * * \pm 0.14$ & $-0.60 \pm 0.33$ & $15.70 * * \pm 0.92$ & $13.20 * * \pm 0.86$ & $0.90 * \pm 0.38$ & $-7.40 * * \pm 1.56$ & $\mathrm{D}$ \\
\hline & \multirow{2}{*}{$\mathbf{C} 2$} & $\mathbf{N}$ & $91.13^{* *} \pm 0.21$ & $7.50 * * \pm 0.46$ & $7.97 * * \pm 1.27$ & $12.47 * * \pm 1.23$ & $6.00 * * \pm 0.49$ & $-12.47 * * \pm 2.11$ & $\mathrm{D}$ \\
\hline & & $\mathbf{L}$ & $83.13^{* *} \pm 0.16$ & $2.50 * * \pm 0.37$ & $-2.02 \pm 1.03$ & $-5.52 * * \pm 0.98$ & $1.00 * \pm 0.42$ & $27.52 * * \pm 1.75$ & $\mathrm{D}$ \\
\hline \multirow{4}{*}{$\begin{array}{l}\text { Plant height, } \\
\text { cm. }\end{array}$} & \multirow{2}{*}{ C1 } & $\mathbf{N}$ & $102.47 * * \pm 0.48$ & $-7.00 * * \pm 1.22$ & $-20.35 * * \pm 3.25$ & $-27.88^{* *} \pm 3.11$ & $-2.00 \pm 1.32$ & $42.54 * * \pm 5.58$ & D \\
\hline & & $\mathbf{L}$ & $85.00 * * \pm 0.41$ & $-5.00 * * \pm 1.04$ & $9.27 * * \pm 2.83$ & $6.00 * \pm 2.66$ & $-2.73 * \pm 1.14$ & $7.60 \pm 4.87$ & $\mathrm{C}$ \\
\hline & \multirow{2}{*}{$\mathbf{C 2}$} & $\mathbf{N}$ & $99.36 * * \pm 0.39$ & $1.03 \pm 1.02$ & $4.68 \pm 2.72$ & $3.68 \pm 2.57$ & $3.57^{* *} \pm 1.11$ & $13.45^{* *} \pm 4.72$ & C \\
\hline & & $\mathbf{L}$ & $91.42 * * \pm 0.63$ & $1.93 \pm 1.41$ & $7.51 \pm 3.94$ & $6.60 \pm 3.79$ & $5.52 * * \pm 1.53$ & $1.44 \pm 6.56$ & $\mathrm{C}$ \\
\hline \multirow{4}{*}{$\begin{array}{l}\text { Number of } \\
\text { spikes / plant }\end{array}$} & \multirow{2}{*}{ C1 } & $\mathbf{N}$ & $6.11^{* *} \pm 0.17$ & $0.73 \pm 0.40$ & $3.58 * * \pm 1.10$ & $2.63 * \pm 1.04$ & $0.39 \pm 0.45$ & $-2.40 \pm 1.87$ & D \\
\hline & & $\mathbf{L}$ & $5.80 \pm 0.12$ & $-0.7 \pm 0.27$ & $0.52 \pm 0.76$ & $0.26 \pm 0.72$ & $-0.29 \pm 0.31$ & $0.75 \pm 1.27$ & $\mathrm{C}$ \\
\hline & \multirow{2}{*}{ C2 } & $\mathbf{N}$ & $6.48 * * \pm 0.15$ & $0.94 * \pm 0.38$ & $5.41^{* *} \pm 1.06$ & $6.64 * * \pm 0.97$ & $0.70 \pm 0.45$ & $-1.54 \pm 1.86$ & $\mathrm{D}$ \\
\hline & & $\mathbf{L}$ & $6.60 * * \pm 0.12$ & $-.0 .07 \pm 0.29$ & $5.62 * * \pm 0.80$ & $4.55^{* *} \pm 0.76$ & $-0.73 * \pm 0.35$ & $-5.09 * * \pm 1.36$ & $\mathrm{D}$ \\
\hline \multirow{4}{*}{$\begin{array}{l}\text { Number of } \\
\text { kernels / } \\
\text { spike }\end{array}$} & \multirow{2}{*}{ C1 } & $\mathbf{N}$ & $29.19 * * \pm 0.63$ & $-4.56 * * \pm 1.48$ & $12.78^{* *} \pm 4.11$ & $9.12 * \pm 3.89$ & $-1.87 \pm 1.63$ & $3.96 \pm 6.95$ & $C$ \\
\hline & & $\mathbf{L}$ & $28.00 * * \pm 0.64$ & $-1.96 \pm 1.62$ & $10.73 * \pm 4.61$ & $8.80 * \pm 4.12$ & $-1.07 \pm 1.83$ & $-7.57 \pm 8.09$ & $\mathrm{D}$ \\
\hline & \multirow{2}{*}{ C2 } & $\mathbf{N}$ & $34.10 * * \pm 0.77$ & $-2.02 \pm 1.83$ & $4.01 \pm 5.07$ & $0.18 \pm 4.78$ & $-0.17 \pm 2.00$ & $21.76 * \pm 8.64$ & $\mathrm{C}$ \\
\hline & & $\mathbf{L}$ & $29.94 * * \pm 0.76$ & $-1.56 \pm 1.83$ & $-6.95 \pm 5.08$ & $-8.20 \pm 4.76$ & $1.29 \pm 2.11$ & $34.26 * * \pm 8.68$ & D \\
\hline \multirow{4}{*}{$\begin{array}{l}\text { 100-kernel } \\
\text { weight, } g \text {. }\end{array}$} & \multirow{2}{*}{ C1 } & $\mathbf{N}$ & $4.85 \pm 0.05$ & $0.21 * * \pm 0.11$ & $0.90 * \pm 0.32$ & $0.62 \pm 0.31$ & $-0.01 \pm 0.12$ & $0.80 \pm 0.53$ & C \\
\hline & & $\mathbf{L}$ & $3.95 * * \pm 0.03$ & $0.40 * * \pm 0.08$ & $0.90 * * \pm 0.23$ & $1.00 * * \pm 0.21$ & $0.11 \pm 0.10$ & $-0.4 \pm 0.40$ & $\mathrm{D}$ \\
\hline & \multirow{2}{*}{$\mathbf{C} 2$} & $\mathbf{N}$ & $3.90 * * \pm 0.03$ & $-0.45 * * \pm 0.06$ & $2.59 * * \pm 0.18$ & $2.40 * * \pm 0.17$ & $-0.13 \pm 0.08$ & $-1.8^{* *} \pm 0.31$ & $\mathrm{D}$ \\
\hline & & $\mathbf{L}$ & $3.60 * * \pm 0.04$ & $0.01 \pm 0.09$ & $1.51^{* *} \pm 0.23$ & $1.47 * * \pm 0.23$ & $-0.22 * \pm 0.09$ & $-1.4 * * \pm 0.39$ & $\mathrm{D}$ \\
\hline \multirow{4}{*}{$\begin{array}{c}\text { Biological } \\
\text { yield / plant, } \\
\text { g. }\end{array}$} & \multirow{2}{*}{ C1 } & $\mathbf{N}$ & $39.33 * * \pm 1.20$ & $-2.00 \pm 2.70$ & $0.05 \pm 7.58$ & $-4.52 \pm 7.23$ & $-1.03 \pm 3.01$ & $26.10 * \pm 12.65$ & $C$ \\
\hline & & $\mathbf{L}$ & $38.35 * * \pm 0.97$ & $-1.00 \pm 2.09$ & $14.59 * \pm 5.83$ & $10.72 \pm 5.70$ & $-0.13 \pm 2.24$ & $-4.2 \pm 9.55$ & $\mathrm{D}$ \\
\hline & \multirow{2}{*}{$\mathbf{C 2}$} & $\mathbf{N}$ & $40.70 * * \pm 0.60$ & $-10.17 * * \pm 1.36$ & $18.57 * * \pm 3.76$ & $10.73 * * \pm 3.63$ & $-4.00 * * \pm 1.49$ & $25.10 * * \pm 6.27$ & $\mathrm{C}$ \\
\hline & & $\mathbf{L}$ & $30.79 * * \pm 0.76$ & $-3.20 \pm 1.83$ & $25.83 * * \pm 5.10$ & $12.56 * * \pm 4.76$ & $-1.27 \pm 2.09$ & $24.90 * * \pm 8.73$ & C \\
\hline \multirow{4}{*}{$\begin{array}{l}\text { Grain yield/ } \\
\text { plant, } g .\end{array}$} & \multirow{2}{*}{ C1 } & $\mathbf{N}$ & $10.65 * * \pm 0.41$ & $0.81 \pm 1.01$ & $2.93 \pm 2.71$ & $2.19 \pm 2.61$ & $1.38 \pm 1.07$ & $2.51 \pm 4.61$ & C \\
\hline & & $\mathbf{L}$ & $10.57 * * \pm 0.25$ & $-1.31 * \pm 0.62$ & $-2.26 \pm 1.71$ & $-3.30 * \pm 1.59$ & $-0.84 \pm 0.69$ & $9.70 * * \pm 2.97$ & $\mathrm{D}$ \\
\hline & \multirow{2}{*}{$\mathbf{C 2}$} & $\mathbf{N}$ & $11.93 * * \pm 0.48$ & $1.90 \pm 1.08$ & $15.56 * * \pm 3.03$ & $11.57 * * \pm 2.87$ & $3.34 * * \pm 1.18$ & $-9.21 \pm 5.08$ & $\mathrm{D}$ \\
\hline & & $\mathbf{L}$ & $9.13^{* *} \pm 0.27$ & $-0.77 \pm 0.66$ & $7.64 * * \pm 1.87$ & $3.38 * \pm 1.71$ & $0.09 \pm 0.75$ & $7.16^{*} \pm 3.24$ & $\mathrm{C}$ \\
\hline
\end{tabular}

\footnotetext{
${ }^{*} p<0.05, * * p<0.01 . \quad \mathrm{C}=$ Complementary $\quad \mathrm{D}=$ Duplicate
} 
Additive gene effect (d) was positive and significant or highly significant for days to heading in cross 1 under normal sowing and cross 2 under the two sowing dates, number of spikes/plant in cross 2 under normal conditions, 100-kernel weight in cross 1 under normal and late sowings, indicating the significant contribution of additive gene effect in the inheritance of these traits and the potential for obtaining further improvement of these traits by selection using pedigree method. On the other hand, negative and significant or highly significant values were found for plant height in cross 1 under the two sowing dates, 100-kernel weight in cross 2 under normal sowing conditions, number of kernels/spike in cross 1 under normal conditions, biological yield/plant in cross 2 under normal conditions and grain yield/plant in cross 1 under late sowing conditions. Similar results were reported by El-Aref et al. (2011), Amin (2013), Hamam (2014), El-Hawary (2016) and Abd El-Rady (2018). Kumar et al. (2017) observed that the additive gene effect was significantly negative for 1000kernel weight.

Dominance gene action (h) was positive and significant or highly significant for days to heading in the two crosses under normal sowing and cross 1 under late sowing conditions, plant height in cross 1 under late sowing conditions, number of spikes/plant in the two crosses under normal condition and cross 2 under late sowing date conditions, number of kernels/spike in cross 1 under the two sowing dates,100kernel weight in the two crosses under both environments, biological yield/plant in the two crosses under late sowing conditions and cross 2 under normal and grain yield/plant in cross 2 under the two sowings (Table 5). Meanwhile, negative and highly significant effects were recorded for plant height in cross 1 under normal sowing conditions. These results show the great importance of the dominance gene effects in the inheritance of these characters. The negative sign for dominance effects indicates that the alleles responsible of less value for these traits were dominant over the alleles controlling high value. Amin (2013) and Abd El-Rady (2018) reported a negative sign for dominance for 100-kernel weight under late sowing date. On the other hand, significant dominance and additive gene effects were important in the inheritance of days to heading in the two crosses under normal sowing, plant height in cross 1 under the two sowing dates, number of spikes/plant in cross 2 under normal sowing, number of kernels/spike in cross 1 under normal sowing, 100-kernel weight in the two crosses under normal sowing and cross 1 under late sowing conditions and biological yield/plant in cross 2 under normal sowing. The dominance gene effects were higher than additive ones, indicating that both dominance and additive gene effects were important in inheritance of those traits. Also, selecting for desirable characters may be practiced in early generations but it would be effective in the late ones when dominant effect diminished. These results agreed with those obtained by El-Aref et al. (2011), Zaazaa et al. (2012), Abd El Rahman (2013), Amin 
(2013), Hamam (2014), El-Hawary (2016), Kumar et al. (2017) and Abd El-Rady (2018).

The additive $x$ additive (i) (Table 5) type of epistatic gene effects were positive and significant or highly significant for days to heading in cross 2 under normal sowing and cross 1 under late sowing, plant height in cross 1 under late sowing, number of spikes/plant in the two crosses under normal sowing and cross 2 under late sowing date, number of kernels/spike in cross 1 under the two sowing dates, 100-kernel weight in the two crosses under late sowing and cross 2 under normal sowing, biological yield/plant and grain yield/plant in cross 2 under the two sowing dates, reporting that these traits have increasing genes and selection for improvement could be effective. These results are in accordance with the findings of Moussa (2010), El-Aref et al. (2011), Koumber and El-Gammaal (2012), Hamam (2014), Kumar et al. (2017) and Abd El-Rady (2018). However, negative and significant or highly significant values of additive $\times$ additive gene effects were reported for days to heading in cross 2 under late sowing date, plant height in cross 1 under normal sowing date and grain yield/plant in cross 1 under late sowing date. These results the showed dispersion of alleles in parents. Therefore, selection is of no use in early segregating generations because there is no additive genetic effect to be fixed in these traits. Similar results were obtained by Amin (2013) and Hamam (2014) and Abd El-Rady (2018). Negative additive $\times$ additive gene effects were recorded for plant height, number of spikes/plant, biomass and grain yield/plant (Akhtar and Chowdhry, 2006).

Data concerning the epistatic gene effects, additive $x$ dominance $(j)$, in Table 5 , had different values and were positive and significant and/or highly significant for days to heading in the two crosses under the two sowing dates, plant height in cross 2 under the two environments and grain yield/plant in cross 2 under normal sowing conditions. As additive $\times$ dominance epistasis tends to segregate in next generations, it would be better to delay selection to later generations with increased homozygosity, where additive and additivexadditive variances are prevailing. These results are in harmony with those obtained by Amin (2013), Hamam (2014), Kumar et al. (2017) and Abd El-Rady (2018). Negative and significant or highly significant values were reported for plant height in cross 1 under late sowing date, number of spikes/plant in cross 2 under late sowing date, 100-kernel weight in cross 2 under late sowing date and biological yield/plant in cross 2 under normal sowing date. These results showed that the inheritance of these characters were effective by duplication effect of epistatic gene.

The dominance $\times$ dominance $(I)$ gene interactions (Table 5) were significant or highly significant and positive for days to heading in cross 2 under late sowing, 
plant height in cross 1 and cross 2 under normal sowing, number of kernels/spike in cross 2 under the two sowing dates, biological yield/plant in cross 1 under normal sowing date and in cross 2 under both sowing dates and grain yield/plant in the two crosses under late sowing date conditions. These results confirmed that importance of dominance $\times$ dominance gene action in the genetic system controlling these traits and selection should be effective in late generations. Significant or highly significant and negative dominance $x$ dominance $(I)$ gene interactions were obtained for days to heading in cross 1 under late sowing date and cross 2 under normal sowing date, number of spikes/plant in cross 2 under late sowing date and 100-kernel weight in cross 2 under the two environments, indicating their reducing effect in the expression of these traits and there is no breeding importance in proceeding generations. These results are in accordance with those reported by Akhtar and Chowdhry (2006), El-Aref et al. (2011), Koumber and El-Gammaal (2012), Amin (2013), Hamam (2014), Kumar et al. (2017) and Abd El-Rady (2018).

This type of epistasis was constructed as complementary when dominance $(\mathrm{h})$ and dominance $\times$ dominance $(I)$ gene effects have same sign and duplicate epistasis when the sign was different. The results in (Table 5) showed that duplicate epistasis was prevailing for all studied characters in the two crosses and both environments, except for days to heading in cross 1 under normal sowing, plant height in the two crosses under late sowing and cross 2 under normal sowing, number of spikes/plant in cross 1 under late sowing, number of kernels/spike in the two crosses under normal sowing, 100-kernel weight in cross 1 under normal sowing date, biological yield/plant in the two crosses under normal sowing and cross 2 under late sowing and grain yield/plant in cross 2 under late sowing and in cross 1 under the normal sowing, where complementary epistasis prevailed, indicating that duplicate epistasis was greater in importance than complementary epistasis for most traits. As non-additive effects were higher than additive effects in most of the studied traits, intensive selection through later generations is recommended to improve these traits. Also, the possibility of obtaining desirable segregates through inter-mating in early segregations require breaking undesirable linkage adopting recurrent selection for handling the above crosses for rapid improvement. These results are in accordance with those reported by Saint Pierre et al. (2010), Yadav and Singh (2011), Amin (2013), Hamam (2014) and El-Hawary (2016).

\section{Inbreeding depression, heterosis and potence ratio:}

Percentages of inbreeding depression, heterosis over mid-parents and better parent and potence ratio are presented in Table 6. Positive significant or highly significant heterosis over . 
Table 6. Heterosis, inbreeding depression (I.D\%), potence ratio (P.R\%), components of variation, heritability percentage in broad $\left(h^{2} b\right)$ and narrow $\left(h^{2} n\right)$ senses and expected genetic advance (G.S) of two bread wheat crosses for seven studied traits under normal $(\mathrm{N})$ and late sowing $(\mathrm{L})$ conditions.

\begin{tabular}{|c|c|c|c|c|c|c|c|c|c|c|c|c|c|}
\hline \multirow{2}{*}{ Trait } & \multirow{2}{*}{ Cross } & \multirow{2}{*}{$\begin{array}{c}\text { Sowing } \\
\text { date }\end{array}$} & \multicolumn{2}{|c|}{ Heterosis (\%) } & \multirow{2}{*}{ I.D \% } & \multirow{2}{*}{$\begin{array}{c}\text { P.R } \\
\%\end{array}$} & \multicolumn{3}{|c|}{ Components of variation } & \multirow{2}{*}{$H / D^{1 / 2}$} & \multicolumn{2}{|c|}{ Heritabbility } & \multirow{2}{*}{ G.S\% } \\
\hline & & & M.P & B.P & & & $\mathbf{H}$ & D & $\mathbf{E}$ & & $\mathbf{h}^{2} \mathbf{b}$ & $h^{2} n$ & \\
\hline \multirow{4}{*}{$\begin{array}{l}\text { Days to } \\
\text { heading }\end{array}$} & \multirow{2}{*}{$C_{1}$} & $\mathbf{N}$ & $1.10 * *$ & $5.75^{* *}$ & $2.23 * *$ & 0.29 & 2.93 & 2.94 & 1.73 & 1.00 & 55.95 & 37.34 & 1.70 \\
\hline & & $\mathbf{L}$ & $2.89 * *$ & $4.71^{* *}$ & $6.74 * *$ & 1.67 & 0.10 & 4.51 & 2.06 & 0.15 & 52.41 & 51.83 & 2.68 \\
\hline & \multirow{2}{*}{$C_{2}$} & $\mathbf{N}$ & $-2.62^{* *}$ & $-1.06^{* *}$ & $2.01^{* *}$ & 1.66 & 3.22 & 15.30 & 2.05 & 0.49 & 78.50 & 70.13 & 4.92 \\
\hline & & $\mathbf{L}$ & $4.09 * *$ & $5.95 * *$ & $6.60 * *$ & 2.33 & 2.25 & 6.22 & 2.07 & 0.60 & 63.96 & 54.17 & 3.22 \\
\hline \multirow{4}{*}{$\begin{array}{l}\text { Plant } \\
\text { height }\end{array}$} & \multirow{2}{*}{$C_{1}$} & $\mathbf{N}$ & 7.89** & $2.52 *$ & 0.45 & 1.51 & 89.33 & 26.13 & 16.05 & 1.85 & 68.80 & 25.39 & 3.66 \\
\hline & & $\mathbf{L}$ & $3.70 * *$ & 1.10 & 7.14** & 1.44 & 43.45 & 24.35 & 15.51 & 1.34 & 59.76 & 31.58 & 2.75 \\
\hline & \multirow{2}{*}{$C_{2}$} & $\mathbf{N}$ & 0.96 & -1.44 & $5.43 * *$ & 0.40 & 54.03 & 13.74 & 14.08 & 1.98 & 59.15 & 19.94 & 2.43 \\
\hline & & $\mathbf{L}$ & 0.97 & $-2.73^{*}$ & $4.31^{* *}$ & 0.25 & 36.63 & 116.28 & 21.77 & 0.56 & 75.56 & 65.27 & 13.88 \\
\hline \multirow{4}{*}{$\begin{array}{c}\text { Number of } \\
\text { spikes/ } \\
\text { plant }\end{array}$} & \multirow{2}{*}{$C_{1}$} & $\mathbf{N}$ & $15.26^{* *}$ & $9.12^{* *}$ & $16.30^{* *}$ & 2.80 & 1.79 & 5.98 & 2.78 & 0.55 & 55.29 & 48.09 & 40.44 \\
\hline & & $\mathbf{L}$ & $4.43 * *$ & $0.81^{* *}$ & $7.20 * *$ & 1.23 & 0.29 & 4.07 & 1.11 & 0.27 & 65.50 & 63.22 & 40.27 \\
\hline & \multirow{2}{*}{$\mathrm{C}_{2}$} & $\mathbf{N}$ & $9.59 * *$ & $6.41^{* *}$ & $26.36^{* *}$ & 3.21 & 1.86 & 1.74 & 3.51 & 1.03 & 27.60 & 18.00 & 12.59 \\
\hline & & $\mathbf{L}$ & $4.09 * *$ & $5.22 * *$ & $18.91 * *$ & 1.61 & 0.23 & 2.96 & 1.74 & 0.28 & 46.98 & 45.21 & 25.56 \\
\hline \multirow{4}{*}{$\begin{array}{l}\text { Number of } \\
\text { kernels/ } \\
\text { spike }\end{array}$} & \multirow{2}{*}{$C_{1}$} & $\mathbf{N}$ & $11.11^{* *}$ & 2.70 & $20.18 * *$ & 1.36 & 36.19 & 99.02 & 31.79 & 0.60 & 64.81 & 54.80 & 36.76 \\
\hline & & $\mathbf{L}$ & $6.52 * *$ & 3.38 & $11.03^{* *}$ & 2.15 & 1.42 & 53.93 & 64.64 & 0.16 & 29.71 & 29.32 & 20.69 \\
\hline & \multirow[b]{2}{*}{$\mathrm{C}_{2}$} & $\mathbf{N}$ & $10.15^{* *}$ & $4.99 * *$ & $17.92^{* *}$ & 2.07 & 82.28 & 126.56 & 48.43 & 0.81 & 63.39 & 47.84 & 33.24 \\
\hline & & $\mathbf{L}$ & $3.70^{*}$ & $-4.37^{*}$ & $18.91^{* *}$ & 0.44 & 20.48 & 121.14 & 64.91 & 0.41 & 50.30 & 46.38 & 36.47 \\
\hline \multirow{4}{*}{$\begin{array}{c}\text { 100-kernel } \\
\text { weight }\end{array}$} & \multirow{2}{*}{$\mathrm{C}_{1}$} & $\mathbf{N}$ & $5.44 * *$ & $1.24 * *$ & $11.82 * *$ & 1.31 & 0.14 & 0.88 & 0.12 & 0.40 & 79.28 & 73.33 & 24.13 \\
\hline & & $\mathbf{L}$ & $-2.26 * *$ & $-8.40 * *$ & $8.25^{* *}$ & -0.34 & 0.09 & 0.12 & 0.16 & 0.88 & 34.72 & 25.00 & 6.39 \\
\hline & \multirow{2}{*}{$\mathrm{C}_{2}$} & $\mathbf{N}$ & $4.12 * *$ & $-2.67 * *$ & $18.02 * *$ & 0.59 & 0.01 & 0.12 & 0.10 & 0.16 & 39.50 & 39.03 & 8.18 \\
\hline & & $\mathbf{L}$ & $0.90 * *$ & $-4.48^{* *}$ & $10.27 * *$ & 0.16 & 0.27 & 0.32 & 0.07 & 0.92 & 75.88 & 53.33 & 16.72 \\
\hline \multirow{4}{*}{$\begin{array}{c}\text { Biological } \\
\text { yield/ } \\
\text { plant }\end{array}$} & \multirow[b]{2}{*}{$C_{1}$} & $\mathbf{N}$ & $11.06 * *$ & $8.52 * *$ & $14.25^{* *}$ & 4.72 & 24.22 & 430.36 & 105.44 & 0.24 & 67.91 & 66.05 & 62.44 \\
\hline & & $\mathbf{L}$ & $9.49 * *$ & $7.21^{* *}$ & $14.01^{* *}$ & 4.46 & 70.10 & 317.83 & 34.46 & 0.47 & 83.66 & 75.35 & 57.78 \\
\hline & & $\mathbf{N}$ & $16.17^{* *}$ & $3.05 * *$ & $27.67^{* *}$ & 1.27 & 36.87 & 101.62 & 20.87 & 0.60 & 74.20 & 62.81 & 28.59 \\
\hline & $\mathbf{C}_{2}$ & $\mathbf{L}$ & $36.18^{* *}$ & $29.36 * *$ & $38.34 * *$ & 6.86 & 22.62 & 119.34 & 64.87 & 0.44 & 50.17 & 45.83 & 34.99 \\
\hline & & $\mathbf{N}$ & $6.19 * *$ & 1.38 & $16.14 * *$ & 1.31 & 50.61 & 32.71 & 9.66 & 1.24 & 75.02 & 42.30 & 50.88 \\
\hline Grain & $C_{1}$ & $\mathbf{L}$ & $9.62^{* *}$ & $5.01^{* *}$ & $10.93 * *$ & 2.19 & 12.27 & 7.96 & 6.57 & 1.24 & 51.73 & 75.35 & 58.78 \\
\hline & & $\mathbf{N}$ & 29.81** & $17.24 * *$ & $31.48^{* *}$ & 2.78 & 8.49 & 64.40 & 16.54 & 0.36 & 67.48 & 63.31 & 77.97 \\
\hline & $\mathbf{C}_{2}$ & $\mathbf{L}$ & $40.69 * *$ & $30.07^{* *}$ & $38.07^{* *}$ & 4.98 & 3.33 & 12.67 & 9.22 & 0.51 & 43.75 & 38.67 & 35.31 \\
\hline
\end{tabular}

$*_{p}<0.05, * *_{p}<0.01$. M.P $=$ Heterosis over mid parents

B.P $=$ Heterosis over better parent 
mid-parent and better parent values were observed for all studied characters in the two crosses under both conditions, except over mid-parents for days to heading in cross 2 under normal sowing, plant height in cross 2 under the two sowing dates and 100- kernel weight in cross 1 under late sowing and over better parent for days to heading in cross 2 under normal sowing, plant height in cross 1 under late sowing and cross 2 under both sowing dates, number of kernels/spike in cross 1 under both sowing dates and cross 2 under late sowing, 100-kernel weight in cross 1 under late sowing and in cross 2 under both sowing dates and grain yield/plant in cross 1 under normal sowing. These results are in accordance with those found by Abd El-Rahman (2013), Hamam (2014), Abd El-Hamid and El-Hawary (2015), El-Hawary (2016) and Abd El-Rady (2018). Better parent heterosis values for grain yield/plant were highly significant and positive in the two crosses, indicating that they could be considered promising crosses in the wheat breeding program to produce hybrid wheat.

Inbreeding depression measured as reduction in performance of $F_{2}$ generation relative to $F_{1}$ is presented in (Table 6 ). Results showed significant or highly significant positive inbreeding depression values for all studied characters under both normal and late sowing dates, except for plant height in cross 1 under normal sowing. These results are expected because the expression of heterosis in $F_{1}$ will be reduced in $F_{2}$ generation due to selfing and starting homozygosity. These results are in close agreements with those of Yadav and Singh (2011), Hamam (2014), Said (2014) and El-Hawary (2016).

Potence ratio (Table 6) refers to over dominance in the two crosses at both sowing dates for most studied traits, where its values exceeded unity. Meanwhile, potence ratio values for days to heading in cross 1 under normal sowing, plant height in cross 2 under both sowing dates, number of kernels/spike in cross 2 under late sowing and 100-kernel weight in the two crosses under late sowing and in cross 2 under normal sowing were less than unity, indicating partial dominance for these characters. Similar findings were obtained by Yadav and Singh (2011), Amin (2013), Hamam (2014), Kumar et al. (2017) and Abd El-Rady (2018).

\section{Genetic components of variance:}

The components of genetic variance, additive (D) and dominance $(H)$ gene effects in Tables (6), revealed that dominance variance component was higher than the additive one for plant height in the two crosses under normal and cross 1 under late sowing, number of spikes/plant in cross 2 under normal sowing and grain yield/plant in cross 1 under both sowing dates, indicating that dominance gene effect play an important role in inheritance of these characters and selection may be effective in later segregating generations. On the other hand, additive gene effects 
were more important in the genetic system controlling the remaining traits, suggesting the effectiveness of selection in early segregating generations to isolate lines characterized with high grain yield under late sowing, indicating heat stress tolerance. Similar results were obtained by El-Aref et al. (2011), Amin (2013), Hamam (2014), El-Hawary (2016) and Abd El-Rady (2018).

The average degree of dominance $(H / D)^{0.5}$ given in (Table 6) was less than unity in most traits, except days to heading in cross 1 under normal sowing, plant height in the two crosses under normal sowing and cross 1 under late sowing, number of spikes/plant in cross 2 under normal sowing and grain yield/plant in cross 1 under both sowing dates. These results confirm the role of partial dominance gene effects in controlling these characters and selection for these traits might be more effective in early generations. Meanwhile, the remaining traits which had degree of dominance more than unity, indicate that over dominance gene effects are controlling such traits and selection should be delayed to later generations for improving these traits. These results indicated that the genetic systems of these characters under the two conditions are controlled by additive and non-additive gene effects. These results are in accordance with those reported by Farshadfar et al. (2008), Khattab (2009), El-Aref et al. (2011), Amin (2013), Hamam (2014), El-Hawary (2016) and Abd El-Rady (2018).

\section{Heritability in broad and narrow-senses and genetic advance:}

Heritability estimates in broad and narrow-senses and genetic advance are presented in (Table 6). The heritability values in broad sense were moderate to high for all studied traits in the two crosses under both sowing dates, except number of spikes/plant in cross 2 under normal sowing and number of kernels/ spike in cross 1 under late sowing. They ranged from $34.72 \%$ for 100-kernel weight in cross 1 under late sowing to $83.66 \%$ for biological yield/plant in cross 1 under late sowing, indicating that most of the phenotypic variability was due to genetic effects and possibility these traits could be improve through selection. The difference between $\mathrm{h}^{2} \mathrm{~b}$ and $h^{2} n$ indicated that the dominance was found in the genetic of these traits. Heritability values are categorized as high (60\% and above), moderate (30-60\%) and low (0-30\%) as stated by Robinson et al. (1949). Narrow sense heritability values were moderate to high in most traits, except for plant height in the two crosses under both sowing dates, number of spikes /plant in cross 2 under normal sowing, number of kernels/spike in cross 1 under late sowing and 100-kernel weight in cross 1 under late sowing, indicating that these traits were greatly affected by additive and non additive effects and there is appreciable amount of heritable variation. Meanwhile, selection for the remaining traits which had low narrow sense heritability estimates 
will be difficult because of problem-causing environmental influence. These results are in accordance with those reported by El-Sayed and El-Shaawawy (2006), El-Aref et al. (2011), Amin (2013), El- Hawary (2016) and Abd El-Rady (2018).

Genetic advance as percent of mean is classified as high ( $>20 \%)$, moderate $(10-20 \%)$ and low $(<10 \%)$ as stated by Johnson et al. (1955). Based on this, the expected genetic advance (G.S) as percent of $F_{2}$ average (Table 6) was moderate to high in the two crosses under both sowing dates, except for days to heading in the two crosses under both sowing dates, plant height in the two crosses under normal sowing and in cross 1 under late sowing and 100-kernel weight in cross 1 under late sowing and cross 2 under normal sowing. This indicates the possibility of doing selection in early generations to enhance achieving high yielding genotypes. Meanwhile, the remaining traits, which showed low values of expected genetic advance, suggest the role of environmental factors and dominance gene action in inheritance system of these traits. Similar results were reported by El-Aref et al. (2011), Amin (2013), Hamam (2014), El-Hawary (2016) and Abd El-Rady (2018).

\section{REFERENCES}

1. Abd El-Hamid E.A.M and M.N.A. El-Hawary. 2015. Generation mean analysis for some quantitative traits in bread wheat. Proc. The $5^{\text {th }}$ Field Crops Conf., FCRI, ARC, Giza, Egypt, 93, 2(A), 2015.

2. Abd El-Rady A. G. 2018. Genetic analysis of some agronomic traits in two bread wheat crosses under heat stress conditions. J. Plant Production, Mansoura Univ., 9 (1): 21-28.

3. Abd El-Rahman, Magda, A. 2013. Estimation of some genetic parameters through generation mean analysis in three bread wheat crosses. Alex. J. Agric. Res., 58(3): 183-195.

4. Akhtar, N. and M.S. Chowdhry. 2006. Genetic analysis and some other quantitative traits in bread wheat. Int. J. Agric. and Biol., 8 (4): 523-527.

5. Amin, I.A. 2013. Genetic behaviour of some agronomic traits in two durum wheat crosses under heat stress. Alex. J. Agric. Res., 58 (1): 53-66.

6. Bayoumi, T.Y.; H. Eid, Manal and E.M. Metwali. 2008. Application of physiological and biological indices as a screening technique for drought tolerance in wheat genotypes .African J. Biotechn., 7 (14): 2341-2352.

7. Bilgin, O.; İ. Kutlu and A. Balkan. 2016. Gene effects on yield and quality traits in two bread wheat ( $T$. aestivum L.) crosses. Int. J. Crop Sci. and Tech., 2 (1): 1-10.

8. El-Aref, Kh.A.O.; A.M. Tammam; M.M. Ibrahim and Y.S.I. Koubisy. 2011. Generation mean analysis in bread wheat under drought conditions. Egypt. J. Appl. Sci., 26 (2): 187-208. 
9. El-Gizawy, N.Kh.B. 2009. Effect of planting date and fertilizer application on yield of wheat under no till system. World J. Agric. Sci., 5 (6):777-783.

10. El-Hawary, M.N.A. 2016. Estimation of some genetic parameters using the sixpopulation mean analysis in four bread wheat crosses. Proc. The $6^{\text {th }}$ Field Crops Conf., FCRI, ARC, Giza, Egypt, 22-23 Nov. 2016.

11. El-Maghraby, O.M.; A.F. Khalaf; A. Abdo,Fatma and M. Sabra, Hosna. 2016. Effect of sowing date on yield and yield components of bread wheat cultivars under environmental conditions of Sohag region. J. Env. Studies, 15: 19-30.

12. El-Sayed, E.A.M. and G.A. El-Shaawawy. 2006. Genetical studies on yield and some agronomic characters in some bread wheat (Triticum aestivum L.) crosses. J. Agric. Sci. Mansoura Univ., 31(8): 4901-4914.

13. Farshadfar, E.; M. Ghanadha; M. Zahravi and J. Sutka. 2001. Generation mean analysis of drought tolerance in wheat (Triticum aestivum L.). Acta-Agron-Hung., 49 (1): 59-66.

14. Farshadfar, E.; S. Mahjouri and M. Aghaee. 2008. Detection of epistasis and estimation of additive and dominance components of genetic variation for drought tolerance in durum wheat. J. Bio. Sci., 8 (3): 598-603.

15. Fischer, R.A. 1986. Physiological limitation to producing wheat in semitropical and tropical environments and possible selection criteria. Proc. Inter. Symp. Wheat for More Tropical Environments, $209-230$.

16. Hamam, K.A. 2014. Genetic analysis of agronomic traits in bread wheat using six parameters model under heat stress. Egypt. J. Agron., 36 (1): 1-18.

17. Hayman, B. I. 1958. The separation of epistatic from additive and dominance variation in generation means. Heredity, 12 (3): 371-390.

18. Jinks, J. L. and R. M. Jones (1958). Estimation of the components of heterosis. Genetics, 43 (2): 223-234.

19. Johnson, H.W.; H.F. Robinson and R.E. Comstock. 1955. Estimates of genetic and environmental variability in soybean. Agron. J., 47 (7): 314-318.

20. Khattab, A.B. 2009. Genetic behavior of some traits of bread wheat (Triticum aestivum L.). J. Agric. Res. Kafr El-Sheikh Univ., 35 (2): 446-479.

21. Koumber, R.M. and A.A. El-Gammaal. 2012. Inheritance and gene action for yield and its attributes in three bread wheat crosses (Triticum aestivum, I.). world J. Agric. Sci., 8 (2): 156-162.

22. Kumar, S.; A. Kumar and J. Kumar. 2017. Genetic analysis for heterotic traits in bread wheat (Triticum aestivum L.) using six parameters model. Elec. J. Pla. Breeding, 8 (1): 206-215. 
23. Mather, K. 1949. Biometrical Genetics. The study of continuous variation. methuen and Co. Ltd.; London.

24. Mather, K. and J.L. Jinks. 1982. In: Biometrical Genetics, third ed. Chapman and Hall Ltd., ISBN-10, 0412228904.

25. Moussa, A. M. 2010. Estimation of epistasis, additive and dominance variation in certain bread wheat (Triticum aestivum, L.) crosses. J. Plant Prod., Mansoura Univ., 1 (12): 1707-1719.

26. Robinson, H. F.; R. E. Comstock and P. H. Harve. 1949. Estimates of heritability and the degree of dominance in corn. Agron. J., 41 (8): 352-359.

27. Said, A.A. 2014. Generation mean analysis in wheat (Triticum aestivum L.) under drought stress conditions. Annals of Agric. Sci., 59 (2): 177-184.

28. Saint Pierre, C.; J. Crossa; Y. Manes and M. P. Reynolds. 2010. Gene action of canopy temperature in bread wheat under diverse environments. Theoretical and Applied Genetics, 120 (6): 1107-1117.

29. Seleiman, M.; M. Ibrahim; S. Abdel-Aal and G. Zahran. 2011. Effect of sowing dates on productivity, technological and rheological characteristics of bread wheat. J. Agron. Crop Sci., 2 (1): 1-6.

30. Tammam, A.M. 2005. Generation mean analysis in bread wheat under different environmental conditions. Minufiya J. Agric. Res., 30 (3): 937-956.

31. Waner, J.N. 1952. A method for estimating heritability. Agron. J., 44 (9): 427430.

32. Yadav, H.K. and S.P. Singh. 2011. Inheritance of quantitative traits in opium poppy (Papaver somniferum L.). Genetika, 43(1): 113-128.

33. Zaazaa, E.I.; M.A. Hagerand and E.F. El-Hashash. 2012. Genetical analysis of some quantitative traits in wheat using six parameters genetic model. AmricanEurasian J. Agric. Environ. Sci., 12 (4): 456-462. 


\title{
تحليل متوسط الأجيال لهجينين من قمح الخبز \\ تحت ظروف الزراعة العادية والمتأخرة
}

\author{
ياسر سيا ابر اهيم قبيصي \\ قسم بحوث القمح ـ معهد بحوث الدحاصيل الحقلية ـ مركز البحوث الزراعية ـ الجيزة - مصر
}

أجريت هذه الدراسة بمحطة البحوث الزراعية بشندويل في ثلاثة مواسم زراعية هي

2016/2015، 2017/2016 و 2018/2017 بغرض دراسة طبيعة الفعل الجيني ونظام التحكم

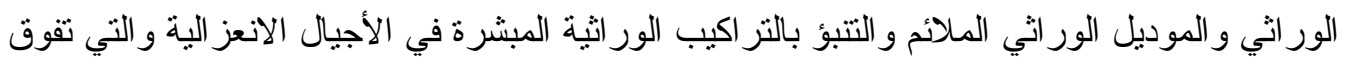

حدود الآباء الداخلة في التهجين وذلك لسبع صفات اقتصادية لهجينين من قمح الخبز (سدس 12 × 11 ×

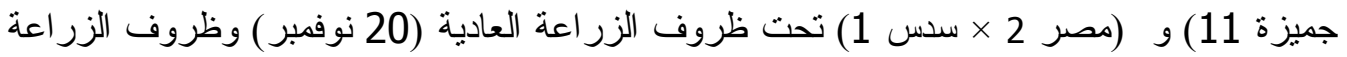
المتأخرة (20 ديسمبر) باستخدام موديل العشائر الست (الأب الأول، الأب الثاني، الجيل الأول، الجيل

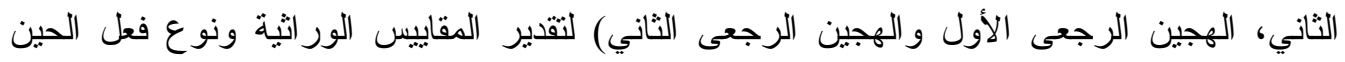

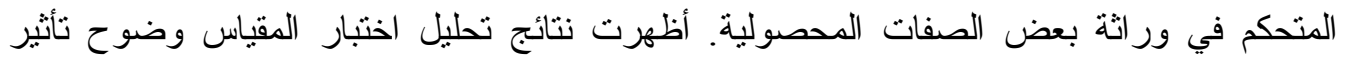

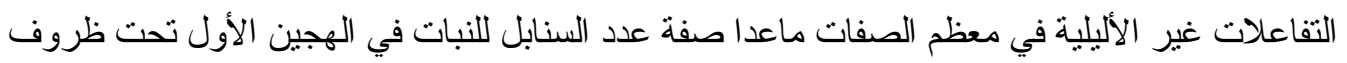

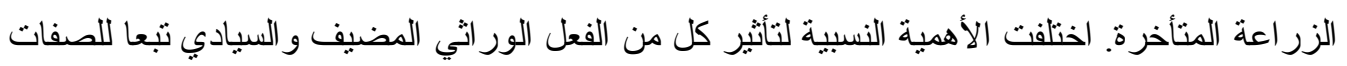

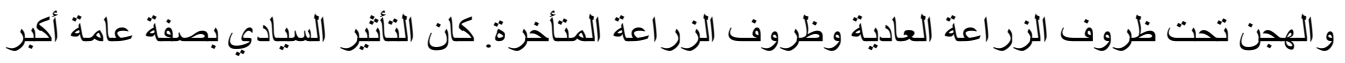

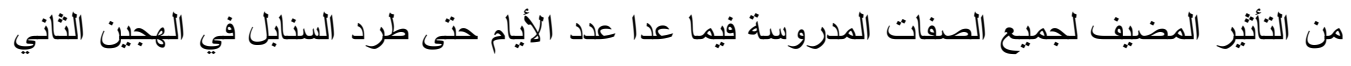

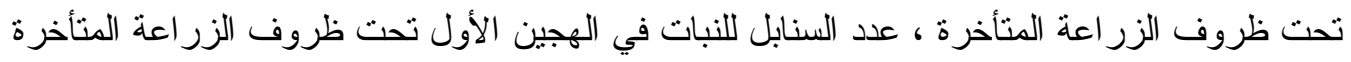

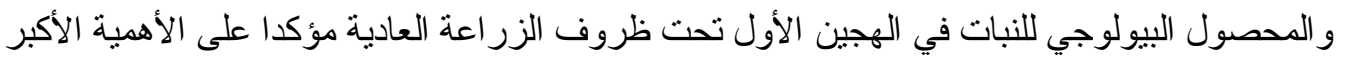

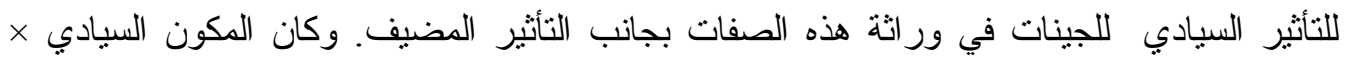
السيادي ذو تأثثر أكبر من تأثثر كل من المضيف × المضيف و المضيف × السيادي في معظم الصفات

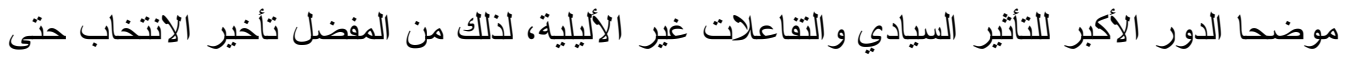

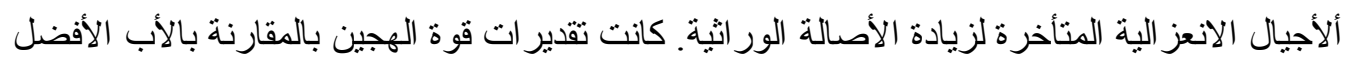

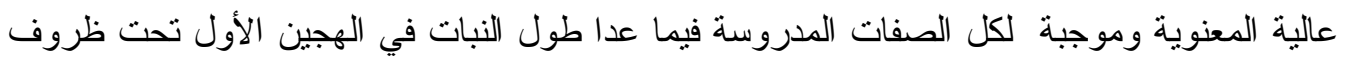
الزر اعة المتأخرة والهجين الثاني تحت ظروف ولية الزر اعة العادية, عدد الحبوب بالسنبلة في الهجين الأول

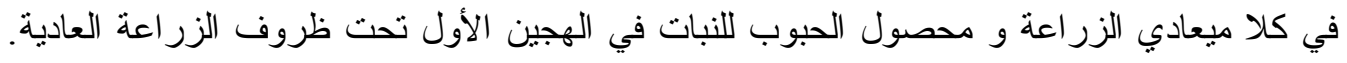

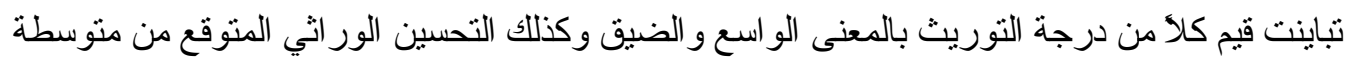

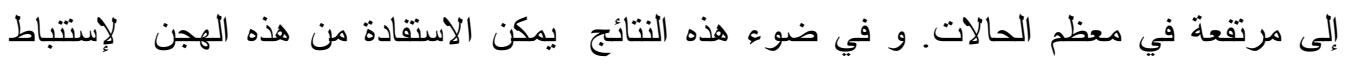
سلالات قمح مبكرة النضج عالية المحصول تحت ظروف فئ ألإجهاد الحر اري (الزر اعة المتأخرة). 

SOWING DATE CONDITIONS 